\title{
Comparison of Antimicrobial Effects of two Different Mouthwashes as A Pre-Procedural Rinse-An Invivo Study
}

\author{
Seba Abraham, Nithin Markose Reji*, Arunima PR and Reejamol MK \\ Deptrtment of Periodontics, PMS College of dental science and Research Vattapara, Trivandrum \\ *Corresponding Author: Nithin Markose Reji, Deptrtment of Periodontics, PMS College of dental science and Research Vattapara, \\ Trivandrum.
}

Received: June 24, 2019; Published: August 16, 2019

DOI: $10.31080 / A S D S .2019 .03 .0616$

\begin{abstract}
Aim: To estimate the antimicrobial effect of $0.2 \%$ Chlorhexidine and HiOra mouth wash in reducing the bacterial load. Objectives:

1. To evaluate the clinical efficiency of $0.2 \%$ chlorhexidine as a pre rinsing mouthwash.

2. To evaluate the clinical efficiency of HiOra mouthwash as a pre rinsing mouthwash.

3. To evaluate the microbial load without use of pre-rinsing mouthwashes.

Materials and Methods: This study is a randomized clinical study in which 45 patients of both sexes within the age range of 20 to 70 from both the genders were selected. Patients were categorized into three groups (group A, B, C) based on random selection. Un-stimulated saliva was collected from all the three groups prior to the procedure. Group A was given normal saline mouth rinse, group B was given $0.2 \%$ Chlorhexidine, and Group C was given herbal mouthwash (HiOra) as a pre procedural rinse. After rinsing with mouthwashes saliva samples are further collected. All the samples collected were sent to the laboratory for analyzing colony forming units (CFU).

Conclusions: Pre-procedural rinse significantly reduce the amount of microbial load of aerosols. chlorhexidine was found to be more effective than herbal mouthwash.

Keywords: Aerosol Production; Colony Forming Unit; Pre-Procedural Rinse; Chlorhexidine; Herbal Mouthwash
\end{abstract}

\section{Introduction}

Dental procedures causes cross contamination in the dental operatory due to aerosolisation of oral microbes during dental procedures which leads to the transmission of infectious agents [1]. Aerosols spawned from patient's mouth measured up to a million bacteria per cubic foot of air [2]. Most of the procedures of the mouth, forms aerosol and splatters which contains bacteria, fungi, protozoa and even viruses [3] These aerosols have association with respiratory infections, ophthalmic infections, skin infections, tuberculosis and hepatitis B [4]. Aerosol and splatter can be produced naturally and artificially in a dental setup, former can be produced from either coughing or sneezing in the dental premise by either patients or practitioners and in the case of latter, it is produced from high speed hand pieces, ultrasonic scalers, and three-way syringes [5]. Aerosols and splatter contains microorganism, which is generated from plaque, calculus, dental materials, and from saliva and blood during dental procedures.

Most dental aerosols have particle size with a diameter of 5 micrometer or less. It can cause both respiratory and other 
infections, based on the penetrating power of the aerosols to the alveoli [6]. Compared to other treatment modalities, use of ultrasonic scalers has been found to create more aerosols and splatters [7].

Majority of dental clinics are having air conditioning system which can be a major harboring source for bacteria and fungi [8]. In addition to the ventilation system, other factors like humidity, temperature and particle size of the aerosols can contribute to the infective contamination of aerosols [9].

Aerosols mainly harbor streptococcus and staphylococcus species.Other infectious agents like mycobacterium tuberculosis have been found in the aerosols generated by the hand piece. There were incidences that reported the presence of legionellaa pneumophila in dental workers [3].

There for many strategies that is done to reduce the bacterial contamination can reduce the cross contamination in the dental office. Several methods like flushing water from the hand piece at the start of each clinical day [10], use of high vacuum suction [11], use of rubber dam [12] and finally the use of pre-procedural rinse of oral cavity with a mouthwash [13] can reduce the cross contamination in the dental office. Snophia., et al. [14] reported that there is substantial increase in aerosol contamination when using ultrasonic devices and high speed hand instrument in dental setup.

Veksler., et al. [15] demonstrated that pre-procedural rinse with $0.2 \%$ chlorhexidine for 60 seconds effectively reduce the quantity of aerobic and facultative bacteria in oral cavity. They eliminate microorganism by destroying the cell walls and constraining the enzymatic activity [16].

Natural herbs have been proven scientifically to be safe and effective against various oral health problems without any side effect till date (Ciancio et al 2008). Herbal mouth rinses are economical and presents with minimum side effects. Natural herbs like triphala, tulsi, jyestiamadh, neem, clove oil, pudina, ajwain have proved to be effective in medicine against various problems like bleeding gums, halitosis, mouth ulcers and preventing tooth decay. Neem has a significant property of antiviral action which is a common ingredient in many of the herbal products. Herbal mouth rinses doesn't have alcohol and sugar like its counterparts.
One such herbal product is $\mathrm{HiOra}^{\circledR}$, a mouthwash known for its antiseptic, antimicrobial, antiplaque, and analgesic property [17]. Hence the aim of the present study was to compare the antimicrobial effect of $0.2 \%$ Chlorhexidine and Herbal mouth wash (HiOra) as a preprocedural rinse in reducing the bacterial load.

\section{Materials and Method}

\section{Materials}

- $\quad$ Saliva samples from patients

- $\quad 0.2 \%$ chlorhexidine gluconate

- Herbal mouth wash (HiOra)

- Normal saline

- $28 g$ Nutrient Agar

- $2.5 \mathrm{ml}$ syringe

- $2 \mathrm{ml}$ vial for collecting saliva

The study was conducted among the outpatients of Department. Of Periodontics, (PMS College Of Dental science and Research). Sample processing and all other laboratory procedures were done at Biogenix institute Trivandrum. This study is a randomized clinical study in which 45 patients of both sexes within the age range of 20 to 70 from both the genders were selected. Patients were categorized into three groups (group A, B, C) based on random selection. Patients who were included had a minimum number of 20 tooth with $80 \%$ visible supra gingival plaque, $10 \%$ visible supra gingival calculus and $30 \%$ of the sites with pocket depth of $5 \mathrm{~mm}$. Patients who had soft or hard tissues lesions, Orthodontic or prosthodontic appliances, carious lesions that required immediate attention, antibiotic therapy within the past three months, history of allergy to CHX or Herbal mouthwashes, underwent oral prophylaxis one month prior to study, Pregnant or lactating patients, systemic condition that may require prophylactic medication for dental treatment were excluded.

The above mentioned 45 subjects were randomly grouped into 3 groups as follows:

Group 1: 15 subjects advised to rinse with normal saline for 1 minute.

Group 2: 15 subjects advised to rinse with10 $\mathrm{ml}$ of $0.2 \%$ chlorhexidine for 1 minutes.

Group 3: 15 subjects advised to rinse with $15 \mathrm{ml}$ Herbal mouth wash with equal dilution with water for 1 minute. 
The subjects included in the study were instructed not to use any chemical plaque control measures 24 hours prior to the procedure. Plaque index, bleeding index, and gingival index were recorded for every patient before the procedure. Un-stimulated saliva was collected from all the three groups prior to the procedure. Subjects were asked to rinse with normal saline, $0.2 \%$ chlorhexine and herbal mouthwash in the respective groups for 1 minutes. Salivary samples were again collected from the test and control groups. All the samples collected was sent to the laboratory for analyzing colony forming units (CFU).

\section{Plate preparation}

The Nutrient Agar media was prepared by dissolving $28 \mathrm{~g}$ Nutrient Agar (HiMedia) in 1000 ml distilled water and sterilized by autoclaving at $121^{\circ} \mathrm{C} 15 \mathrm{lbps}$ for $15 \mathrm{mins}$.

Estimation of colony forming units

- The whole procedure was conducted in a Laminar Air flow hood.

- $\quad$ The saliva samples were vortexed and $10 \mu \mathrm{l}$ from each were swabbed on to Nutrient agar plates. The plates were incubated at $37^{\circ} \mathrm{C}$ for $24-48$ hours in a microbiological incubator.

- After incubation the plates were observed for colony forming units (CFUs). The CFUs were counted using a Digital Colony counter and were expressed in CFUs/ml.

\section{Result}

In this research we have compared three groups of subjects (Group A,Band C) in which group A was given normal saline mouth rinse, group B was given $0.2 \%$ Chlorhexidine, Group $\mathrm{C}$ was given herbal mouthwash (HiOra). The objective of this research was to assess and establish the effectiveness of this mouthwashes as a pre-procedural rinse and to find out the significance of the effectiveness.

All baseline parameters like age, sex, gingival index, periodontal index, bleeding index were recorded.

- $\quad$ Table 1 shows no statistically significant difference between the mean values of Gingival index, Plaque index, Bleeding Index at baseline which was analysed by Kruskal Wallis (Chi square) test with respect to Group A, Group B, and Group C. Hence it was comparable (P value > 0.01).
- Table 2 shows no statistically significant difference in the Mean CFU values among the groups (Group A, Group B, Group C) before rinsing ( $\mathrm{P}$ value $>0.01$ ).

- Table 3 shows no statistically significant difference in the Mean CFU values among the groups (Group A, Group B, Group $\mathrm{C})$ after rinsing ( $\mathrm{P}$ value $>0.01)$.

- $\quad$ Table 4 shows a statistically highly significant difference in CFU values before and after pre- procedural rinse in all the three groups. $(P<0.001)$. The maximum reduction was in group B $(0.2 \%$ Chlorhexidine) compared to group A and $\mathrm{C}$. The least reduction was recorded in group A (normal saline).

\begin{tabular}{|l|c|c|c|}
\hline Mean & Group A & Group B & Group C \\
\hline Gingival index & 2.00 & 1.93 & 1.80 \\
\hline Plaque index & 2.33 & 2.20 & 2.40 \\
\hline Bleeding index & 1.87 & 1.67 & 1.80 \\
\hline
\end{tabular}

Table 1: Pre-procedural evaluation of gingival index, plaque index and bleeding index.

\begin{tabular}{|l|l|l|l|l|}
\hline & Group A & Group B & Group C & P value \\
\hline Mean & 29830.67 & 98660.00 & 100213.33 & 0.88 \\
\hline $\begin{array}{l}\text { Standard } \\
\text { Deviation }\end{array}$ & 9943.3 & 32886.6 & 33404.3 & \\
\hline
\end{tabular}

Table 2: Mean CFU of bacterial load before procedure.

\begin{tabular}{|l|l|l|l|l|}
\hline & Group A & Group B & Group C & P value \\
\hline Mean & 27448.67 & 42104.00 & 85920.00 & 0.043 \\
\hline $\begin{array}{l}\text { Standard } \\
\text { Deviation }\end{array}$ & 9149.3 & 14034.6 & 28640 & \\
\hline
\end{tabular}

Table 3: Mean CFU of bacterial load after rinsing with mouthwash.

\begin{tabular}{|l|c|c|c|}
\hline (CFU/ml) & Group A & Group B & Group C \\
\hline Pre procedural & 29830.67 & 98660.00 & 100213.33 \\
\hline Post procedural & 27448.67 & 42104.00 & 85920.00 \\
\hline Mean difference & 2382.00 & 56556 & 14293.33 \\
\hline
\end{tabular}

Table 4: Mean difference in the CFU before and after rinsing.

\section{Discussion}

Aerosol generated in the dental sets are immensely contaminated with bacteria and can be a health hazard to the 
dentist and patients. Ultrasonic units generates the greatest amount of aerosol and splatter in dentistry [3] which make this procedure one of the chief airborne contaminant in dentistry [18]. The American Dental Association has also vouched for the potential contaminated aerosols during dental procedures. Aerosol were recognised being a potent source for cross-infections like measles, tuberculosis and severe acute respiratory syndrome (SARS) [19]. The potential routes for the spread of infection in a dental office are direct contact with body fluids of an infected patient, contact with environmental surfaces or from contaminated instruments. Use of rubber dams, high-velocity evacuation, brushing before dental visit, pre procedural rinse with mouthwashes are helpful in reducing microbial count in dental aerosols [20].

Preprocedural rinse such as chlorhexidine ( $\mathrm{CHX}$ ) in varying concentrations, are being used to reduce the bacterial contamination before ultrasonic scaling and periodontal surgery. Chlorhexidine with a dilution of $0.2 \%$ is highly effective in inhibiting subgingival plaque formation and hence prevents development of gingivitis [21]. Chlorhexidine is a cationic bisguanide molecule which can strongly bind to the hydroxyappetite, the organic pellicle of the tooth, oral mucosa, salivary protein and bacteria thereby exhibiting superior substantivity in the oral cavity [22]. It is considered to be the most effective anti-plaque and anti-gingivitis agent [19]. It was found to be bactericidal at higher concentration and bacteriostatic at lower concentration.

Apart from chlorhexidine, mouthwashes having active herbal products are also being marketed. AM Khalessi., et al. [23] demonstrated that the effectiveness of the herbal mouth wash helps in minimising the plaque formation. Some of the main ingredient in the herbal mouthwash (HiOra) are Salvadora persica, Terminalia bellerica, Piper betel, Neem, Mulaithi etc. These ingredients have an anti-bacterial property which helps in the release of calcium and fluoride into saliva [24]. Since dental plaque comprises of microorganism of both bacterial and viral in origin, herbal mouthwashes with extracts demonstrates antiviral activity due to presence of neem which would be a better choice [25].

The present study compares the antimicrobial effect of $0.2 \%$ Chlorhexidine and Herbal mouth wash (HiOra) as a preprocedural rinse in reducing the bacterial load based on the estimation of the amount of microorganism present in the saliva before and after the use of mouthwash which was calculated in colony forming units (CFU). The study was conducted on 45 subjects who were divided into three groups of 15 , based on the pre procedural mouth rinse given. Each groups were given normal saline rinse, $0.2 \%$ chlorhexidine and herbal mouthwash respectievely. The potency of the mouthrinse was analysed based on colony forming units (CFU). The differences in CFU before and after rinsing in the three groups were highly significant ( $\mathrm{p}$ value $<0.001$ ). Maximum reduction was obtained in group B $(0.2 \%$ Chlorhexidine) (figure 2) compared to group A (Figure 1) and B (figure 3). The least reduction was obtained in group A (normal saline).

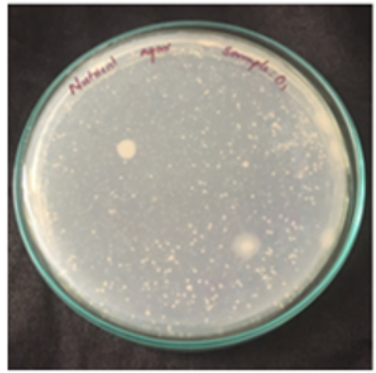

A

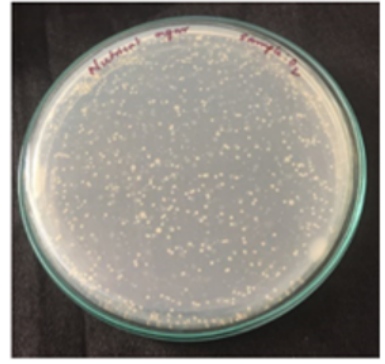

B
Figure 1: Bacterial colony in response to Normal saline rinse A) befor rinsing B) After rinsing.

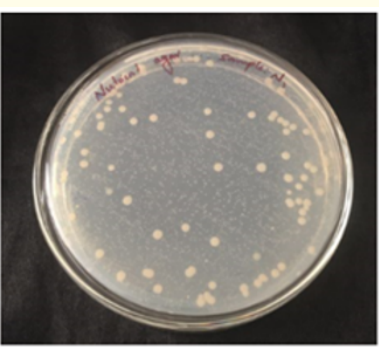

A

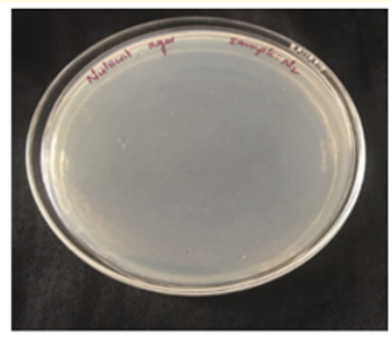

Figure 2: Bacterial colony in response to Chlorhexidine rinse A)befor rinsing B)After rinsing.

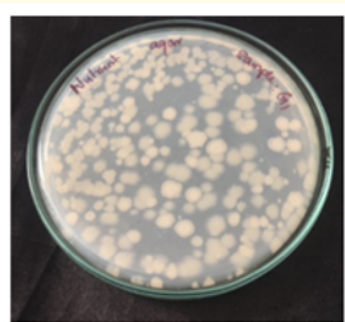

A

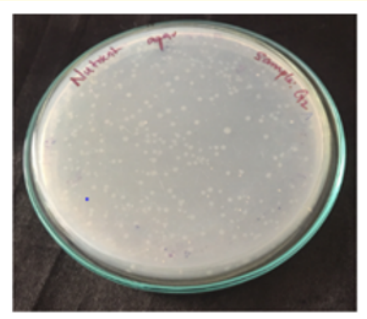

B
Figure 3: Bacterial colony in response to herbal mouthwash rinse A)befor rinsing B)After rinsing. 
The results of the present study is comparable to the previous study by Barnes., et al. [18] who demonstrated that Preprocedural rinse such as chlorhexidine (CHX) in varying concentrations can reduce bacterial contamination. Gupta., et al. also demonstrated the efficacy of preprocedural rinsing with chlorhexidine in reducing the aerosol contamination produced by ultrasonic scaling. Balejo., et al. [26] reported that preprocedural rinsing in periodontally diseased patients showed a discrete effect on bacteremia control. According to Newmann., et al. Chlorhexidine is considered to be the most effective anti-plaque and anti-gingivitis agent.

Herbal mouthwash used in the present study showed reduction in the bacterial count, however chlorhexidine showed marked bacterial reduction potential which was more evident in the study. Bhat., et al. [27] reported beneficial anticariogenic, antiplaque, antibiotic and anti-inflammatory action associated with HiOra mouthwash which is a non-alcoholic preparation made from natural herbs.

Fine., et al. [28] reported that the use of plaque control agents such as chlorhexidine as pre-procedural mouthwash was found to be effective in reducing bacterial count in aerosol, compared to distilled water or saline. In the present study it was found that pre-procedural rinse even with normal saline resulted in some amount of reduction in bacterial burden. However saline was least effective when compared to other two groups. The results of the present study are comparable to the study by Gupta., et al. [4] who compared the efficacy of chlorhexidine, Herbal mouthwash and Water as pre-procedural rinse in the reduction of aerosol contamination.

The present study demonstrated the effectiveness of $0.2 \%$ chlorhexidine in reducing bacterial count when used as a preprocedural rinse over normal saline and herbal mouth wash.

\section{Conclusion}

The bioaerosols generated during the dental procedures increases the risk of infection to dentists and dental personnels. Hence effective control of this contaminated aerosols is warranted. Pre procedural mouthrinses are most beneficial before oral prophylaxis. The present study clearly shows that the preprocedural rinse with anti-microbial mouthwash effectively reduce the bacterial load in oral cavity and thereby reducing aerosol contamination. The present study also shows the efficacy of chlorhexidine over herbal moth was in reduction of bacterial load.

\section{Bibliography}

1. Earnest R and Loesche W. "Measuring harm ful levels of bacteria in dental aerosols". Journal of the American Dental Association 122.12 (1991): 55-57.

2. Miller RL. "Generation of airborne infection by high speed dental equipment". The Journal of the American Society for Preventive Dentistry 6.3 (1976): 14-17.

3. Szymańska J. "Dental bioaerosol as an occupational hazard in a dentist's workplace". Annals of Agricultural and Environmental Medicine 14.2 (2007): 203-207.

4. Gupta G., et al. "Efficacy of preprocedural mouth rinsing in reducing aerosol contamination produced by ultrasonic scaler: A pilot study". Journal of Periodontology 85.4 (2014): 562-568.

5. Mckinley I B Jr and I AudlowhI O. "Hazards of laser smoke during endodontic therapy". Journal of Endodontics 20.11 (1994): 558-559.

6. Pelleu G B., et al. "Reduction of microbial concentration in the air of dental operating rooms. I. High efficiency particulate air filters". Journal of Dentistry 49.2 (1970): 315- 319.

7. Grenier D. "Quantitative analysis of bacterial aerosols in two different dental clinic environments". Applied and Environmental Microbiology 61.8 (1995): 3165- 3168.

8. Burge H Bioaerosols. "Prevalence and health effects in the indoor environment". Journal Allergy and Clinical Immunology 86.5 (1990): 687-701.

9. Burge H A. "Indoor sources for airborne microbes. In Gammage R B, Kaye S V. Eds lndoor Air and Human Health. Chelsea, hlich: Lewis Publisher Inc (1985): 139-148.

10. CarolineL., et al. "Microbial contamination of dental unit waterlines: the scientific arguments". International Dental Journal 48.4 (1998): 359-368.

11. Sagar Abichandani and Ramesh Nadiger. "Cross contamination in dentistry: a comprehensive overview". Journal of Education and Ethics in Dentistry 2.1 (2012): 3-9.

12. Maria LC., et al. "Evaluation of risk of infection through exposure to aerosols splatters in dentistry". American Journal of Infection Control 36.4 (2008): 304-307.

13. Bhanu M and Deepali B. "Infection control and prevention in dentistry". International Journal of Dentistry Advancements 3.3 (2011): 577-582. 
14. Snophia S., et al. "Comparison of efficacy of preprocedural rinsing with chlorhexidine and essential oil mouth in reducing viable bacteria in dental aerosols- a microbiological study". International Journal of Contemporary Dentistry 2.6 (2011): 1-6.

15. Veksler AE., et al. "Reduction of salivary bacteria by pre-procedural rinses with chlorhexidine $0.12 \%$ ". Journal of Periodontology 62.11 (1991): 649-651.

16. Marchetti E., et al. "Efficacy of essential oil mouthwash with and without alcohol: A 3-day plaque accumulation model". Trials 12 (2011): 262.

17. Ranjan Malhotra., et al. "Comparison of the effectiveness of a commercially available herbal mouthrinse with chlorhexidine gluconate at the clinical and patient level". Journal of Indian Society of Periodontology 15.4 (2011): 349-352.

18. Harrel SK., et al. "Aerosol and splatter contamination from the operative site during ultrasonic scaling". Journal of the American Dental Association 129.9 (1998): 1241-1249.

19. Harrel SK and Molinari J. "Aerosols and splatter in dentistry: A brief review of the literature and infection control implications". Journal of the American Dental Association 135.4 (2004): 429-437.

20. Bently C D., et al. "Evaluating spatter and aerosol contamination during dental procedure". Journal of the American Dental Association 125.5 (1994): 579-584.

21. Walsh TF et al. "The effect of irrigation with chorhexidine or saline on plaque vitality". Journal of Clinical Periodontology 22.3 (1995): 262-264.

22. Lyle D. "The Role of Pharmacotherapeutics in the Reduction of Plaque and Gingivitis". Journal of Dental Hygiene 9.6 (2000): 46-50.

23. AM Khalessi., et al. "An in vivo study of the plaque control efficacy of Persica: a commercially available herbal mouthwash containing extracts ofSalvadora persica". International Dental Journal 54.5 (2004) :279-283.

24. Darout IA., et al. "Periodontal status of adult Sudanese habitual users of miswak chewing sticks or toothbrushes". Acta Odontologica Scandinavica 58.1 (2000): 25-30.

25. Mallick A., et al. "Neem leaf glycoprotein activates CD8 (+) T cells to promote therapeutic anti-tumor immunity inhibiting the growth of mouse sarcoma". PLoS One 8.1 (2013): e47434.
26. Balejo RPD., et al. Journal of Applied Oral Science 25.6 (2017): 586-595.

27. Acharya S., et al. "Aerosol contamination in a rural university dental clinic in South India". International Journal of Infection Control 6 (2010): 1.

28. Fine DH., et al. "Efficacy of preprocedural rinse with an antiseptic in reducing viable bacteria in dental aerosol". Journal of Periodontology 63.10 (1992): 821-824.

\section{Volume 3 Issue 9 September 2019 \\ (C) All rights are reserved by Nithin Markose Reji., et al.}

